\title{
Diagnóstico y Tratamiento de las Neoplasias Interepiteliales y de los Carcinomas Cervicales Invasores en el Embarazo
}

\author{
Dres.: Fernando Cardona1, Guillermo Acebedo1, Hugo Quijano2, \\ Francisco Pardo3, Miguel Rodríguez 3 , Jaime Uribe4, Antonio Soto5, \\ Jaime Barrios 5 .
}

Trabajo presentado como "Seminario Co" rrelacionado", en el XII Congreso Mundial de Obstetricia y Ginecología, en San Fran. cisco en 1982.

El diagnóstico y tratamiento de las neoplasias interepiteliales y de los cánceres invasores del cervix en el embarazo será comentado, basados en la experiencia obte. nida en Colombia, en el análisis de 152 casos de esta patología cervical, obtenidos de un total de 10.884 cánceres ginecológicos registrados en diez años.

Universidad Pontificia Bolivariana, Medellín. Instituto Nacional de Cancerología, Bogotá.

3 Universidad Pontificia Javeriana, Bogotá.

4 Universidad de Antioquia, Medellín.

5 Universidad de Cartagena, Cartagena.

\section{DIAGNOSTICO}

\section{La Citología}

En el campo del diagnóstico de las neoplasias interepiteliales y de los cánceres invasivos del cervix durante el embarazo grandes progresos han sido obtenidos /con los nuevos horizontes logrados por medio de la citología exfoliativa.

Hasta hace pocas décadas los ginecoobstetras en general, incluyendo los de Colombia, ten ían el firme convencimiento, por los primeros trabajos efectuados en este campo, de que la citología exfoliativa del exo - y del endocervix durante el embarazo tenía un mínimo valor diagnóstico, y que su credibilidad era muy limitada por causa del fenómeno frecuentemente observado de la presencia de displasias leves o moderadas, que persisten durante todo el embarazo y que en un $50 \%$ de las veces desaparecen espontáneamente después de terminado el puerperio. 
También era conocido que un $20 \%$ de las pacientes presentaban hiperactividad de las células de reserva durante el embarazo y que esta hiperactividad era casi siempre reversible después del parto, $y$ que ella en su lectura en los extendidos daba un cuadro muy semejante a la displasia leve y aún a la moderada (1).

Todo ello trajo como consecuencia la formación de una mentalidad en el gineco-obstetra de que la citología oncológica durante el embarazo tenía una utilidad mínima y sus lecturas sospechosas o positivas debían ser interpretadas con una gran cautela o discresión.

Pero los trabajos efectuados por los citopatólogos en los últimos años han cambiado los anteriores conceptos de la citología cérvico-vaginal durante el embarazo y hoy puede afirmarse que ella es de gran utilidad como elemento diagnóstico en el campo oncológico en las pacientes obstétricas, y así se piensa en Colombia en los centros de Bogotá, Medellín, y Cartagena (2).

Cuando existe una displasia epitelial leve, moderada o severa, o una neoplasia interepitelial o un carcinoma invasivo del cervix durante el embarazo, la citología exfoliativa podrá detectar esta patología cervical en un $97 \%$ de los casos, si son tomadas siempre una muestra del tondo de saco vaginal posterior, otra del exocervix y una tercera del endocervix (3).

Tanto en el embarazo como en los otros estados fisiológicos de la mujer, son marcadas las diferencias observadas en los extendidos cérvico-vaginales de las células epiteliales que se exfolian, y son claras las diferencias morfológicas entre una hiperactividad de las células de reserva y una displasia moderada o severa, o un carcinoma in situ, o un carcinoma invasor del cervix.

Por lo tanto la citología cérvico. vaginal, tomando siempre tres muestras, es una ayuda muy eficaz para el diagnóstico de una neoplasia interepitelial o de un cáncer invasor del cervix en el embarazo, que deberá siempre ser confirmada por el estudio histológico del material obtenido, por medio de la biopsia dirigida, de la biopsia múltiple, o del cono biopsia, como se analizará más adelante.

La lectura de los extendidos cérvico vaginales permite con facilidad distinguir las células normales del epitelio estratificado no cornificado que se encuentra en el exocervix, de las células del epitelio columnar -o epitelio secretor-que son propias del canal endocervical. Cuando las células que se exfolian presentan atipias es posible, en la gran mayoría de los casos; conocer si ellas pertenecen a un epitelio de revestimiento o a un epitelio secretor; por lo tanto, la lectura citológica acompañada de una descripción del tipo de células atípicas que se encuentran, orientará la colposcopia y la toma de la biopsia y aún el modo de efectuar el cono.

El concepto anterior también̨̧es válido en los casos de ectopia y de metaplasia del epitelio del cervix; por ello la citología actual ya no se limita a clasificar el grado de atipia celular que se encuentra, sinó también a describir que tipo de células son las que presentan alteraciones morfológicas; pero esto no es absoluto sino relativo, como son los fenómenos biológicos, pués existen algunos casos en que el epitelio exfoliado es tan inmaduro que no permite precisar su origen.

La citología exfoliativa cérvico vaginal también ayuda al gineco-obstetra cuando estan presentes displasias leves, moderadas o severas, o carcinomas in situ durante el embarazo, pues ella puede indicarle en tomas efectuadas periódicamente, si la lesión existente diagnosticada con anterioridad permanece estática sin agravarse o si ella evoluciona en forma progresiva y llega a definirse como un carcinoma invasor y requiere una conducta diferente; por ello siempre en 
Colombia se controlan estas pacientes con citologías períodicas.

Cuando se efectúa un cono del cervix con fines diagnósticos o con fines terapéuticos durante el embarazo, la citología exfoliativa tomada periódicamente en fechas posteriores también es de gran utilidad, pues en la mayoría de los casos revela si el tejido noeplásico fue extirpado en su totalidad $-y$ en estos casos la citología se torna negativa para células atípicas- o si solo la extirpación fue parcial $y$ quedó en la paciente tejido patológico $-y$ en estos casos la citología seguirá mostrando células con alteraciones morfológicas-.

Todas las consideraciones anteriores permiten afirmar que la citología exfoliativa abre nuevos horizontes en el campo del diagnóstico de las neoplasias interepiteliales y de los carcinomas invasores del cervix en el embarazo, y por lo tanto debe ser un examen que no puede omitirse en una correcta consulta prenatal.

En los hospitales universitarios de Colombia, este procedimiento de la triple toma. citológica cérvico vaginal en la consulta prenatal ha ido aumentando en los últimos años y ya existen centros docentes en donde ella no es omitida en ninguna de las pacientes que consultan durante el embarazo. Pero aún falta mucho para lograr la meta de efectuarla en todas las pacientes, pues existen centros de asistencia social y muchos centros universitarios y consultas particulares, en donde estas tomas citológicas no se hacen por no tener un concepto cierto del valor real de la citología exfoliativa.

Por tener las células de reserva un carácter bipotencial hacia células secretoras o células pavimentosas, en algunos casos la citología atípica de estas ćelulas como también en la citología exfoliativa de las células denominadas inmaduras, no permite definir si la lesión es primitivamente de un epitelio endocervical secretor o de un epitelio exocervical poli-estratificado, y solo será posible sospecharlo citológicamente teniendo en cuenta el punto donde fue tomada la muestra, pero esta limitación en algunas citologías puede ser frecuentemente resuelta por medio de otro procedimiento diagnóstico como lo es la colposcopia.

\section{La Colposcopia}

Con el uso cada vez más frecuente de la colposcopia en la consulta prenatal, grandes progresos se han logrado en el diagnóstico de las neoplasias interepiteliales y aún en los cánceres invasivos del cervix durante el embarazo; con la visión directa y ampliada del cervix empleando soluciones para un mayor contraste, es posible detectar precozmente las metaplasias aún durante el embarazo, en donde la congestión de la mucosa es máxima y el epitelio toma un color rojo violáceo.

Es bien conocido que en el embarazo por acción de los estrógenos y de los progestógenos aumentados, en cerca del $50 \%$ de los casos, el epitelio cilíndrico sobrepasa su habitual límite del orificio externo y progresa hacia el exocervix en el fenómeno conocido como ectopia papilar, y que luego el epitelio estratificado intenta desplazar este epitelio desde la periferia-metaplasia directa o zona de transformación ascendente, -poco frecuente - o mediante la multiplicación de las células de reserva -metaplasia indirecta o multiplicación descendente-, lo más frecuente (4).

Todos estos cambios morfológicos facilmente observados por medio del colposcopio con soluciones de ácido acético $y$ de lugol, pueden persistir durante todo el embarazo y regresar espontáneamente después del parto como ya fue comentado, o en algunos casos persistir $y$ en otras ocasiones evolucionar hacia alteraciones más severas y aún malignas. 
Las imágenes del epitelio blanco - de hiper o para queratosis- solo visibles en el ácido acético, las de leucoplasia, más frecuente la forma rugosa que la plana, las de punteado o "base", las de mosaico, las zonas de transformación atípica, y la observación de las alteraciones vasculares del cervix indicarán al gineco-obstetra en la consulta prenatal el punto que debe de elegir para la toma de la citología dirigida, o de la biopsia dirigida que confirma el diagnóstico sospechado.

Bien es cierto que las lesiones endocervicales de neoplasias interepiteliales, no son detectados por los procedimientos colposcópicos, y de que ellas solo se manifiestan precozmente por medio de la citología, pero ante una citología sospechosa o positiva y una imágen colposcópica negativa, el procedimiento indicado basados en estos datos de la colposcopia, será el efectuar un cono con toma representativa del endocervix.

En casos de carcinoma invasor del cervix durante el embarazo, la colposcopia muy frecuentemente orienta acertadamente en la toma de biopsia representativa y se define pronto la conducta a tomar para un tratamiento correcto $y$ eficaz.

En Colombia este recomendable procedimiento ha logrado implantarse en solo un $15 \%$ en las consultas externas ginecológicas de los Hospitales Universitarios, pero aún falta un gran terreno por recorrer para su uso habitual en las consultas prenatales en donde se efectúa en un número muy reducido de casos.

\section{La Biopsia múltiple}

Cuando la citología exfoliativa cervico-vaginal tomada durante el embarazo, indica la presencia de alteraciones morfológicas que requieren ser definidas, el paso siguiente es el estudio colposcópico que ya se comentó y en su defecto al menos la simple inspección visual del cervix, efectuando la prueba de Schiller con solución yodada; habitualmente practicada en Colombia, y en seguida el estudio histológico del exo y endocervix. Para ello se dispone de dos procedimientos diferentes para el estudio microscópico de estos epitelios y son la biopsia múltiple y el cono.

La biopsia múltiple es la preferida en uno de los tres centros del presente informe y fue efectuada inicialmente orientando sus tomas con solución de ácido acético y posterior solución de lugol (yodado) en aquellos campos en que no tomaban el yodo, es decir cuando existía epitelio sin glucógeno, y efectuando una biopsia más del canal endocervical. En la actualidad en este centro - de Medellín- se continúa efectuando la biopsia múltiple pero orientando sus tomas por medio de la colposcopia.

La experiencia en los 108 casos positivos de este centro en 10 años de estudio ha mostrado su eficacia para un correcto diagnóstico y su mínima morbilidad, durante el embarazo. En este centro solo se utiliza el cono cuando el exocervix se encuentra sin lesión visual a la colposcopia y la citología es fuertemente sospechosa o positiva.

Cuando en el estudio histológico de una o de varias de las tomas de las biopsias múltiples se revela como máxima patología una neoplasia interepitelial, se procede sin más elementos diagnósti$\cos$ al tratamiento que se indicará posteriormente para esta patología, no importando para ello el tiempo de evolución del embarazo, efectuando, eso sí, valoraciones frecuentes citológicas $y$ colposcópicas. Pero cuando existe aunque sea en solo uno de los cortes una invasión franca del estroma, o un carcinoma micro invasor no importa que en otros cortes se observe una neoplasia interepitelial, se considera para efectos del tratamiento como un cáncer invasor del cervix y su tratamiento es el que se 
comentará para esta patología del embarazo, un poco más adelante.

En este centro oncológico de Medellín, se procede a la biopsia múltiple dirigida por el colposcopio y a la toma de una muestra más del canal endocervical tanto en las pacientes embarazadas como en las no embarazadas que requieren este procedimiento diagnóstico. No se pretende con este procedimiento un fin terapéutico sino solamente un fin diagnóstico (5).

\section{Conización del Cervix}

Para un diagnóstico definitivo de si existe o no una neoplasia interepitelial o un carcinoma invasor del cervix durante el embarazo, en los otros dos centros del presente informe efectuado en Colombia, se procede a la conización del cervix, es decir a la biopsia en bloque que tiene como base el exocervix en un diámetro de 3 a 5 milímetros mayor que el de todas las zonas yodo negativas después del aplicado de la solución de lugol, y como vértice el canal endocervical en sus dos tercios inferiores.

Este cono así tomado, permite un estudio histológico casi siempre completo $y$ permite definir si la patología existente es la de una displasia leve, moderada, o severa, o de un carcinoma in situ, o de un carcinoma invasor. $Y$ también logra delimitar si todo el tejido patológico fue extirpado o si solo lo fue parcialmente; es decir si el cono sirvió solo como método diagnóstico o si logró también un efecto terapéutico.

En estos dos centros de Bogotá y Cartagena, se tiene conciencia de la gran morbilidad de este procedimiento diagnóstico efectuado durante el embarazo, en cuanto a las hemorragias postquirurgicas, y los abortos posteriores (o mediados) y los partos prematuros, pero se consideran que todos estos ries- gos son dignos de ser corridos si con el cono se logra definir un acertado diagnóstico, por ejemplo la presencia de uno de los puntos de una lesión invasiva que tendrá un tratamiento radicalmente diferente al efectuado en la patología de la neoplasia interepitelial, como se presentará más adelante.

No existe en Colombia uniformidad de conceptos en cuanto a la biopsia múltiple y el cono cervical como procedimientos diagnósticos durante el embarazo y este mismo fenómeno se observa en la literatura científica (6).

\section{RESULTADOS}

En Colombia con los procedimientos de diagnóstico mencionados, fueron estudiados las pacientes que presentaron neoplasias interepiteliales o carcinomas invasores del cervix durante el embarazo, entre los años de 1972 a 1981, éste estudio aue comprende un período de 10 años fue efectuado en tres centros de diagnóstico y terapia para enfermedades oncológicas ubicados en Bogotá, capital del país con 5.000.000 de habitantes, en Medellín con 2.000.000 de habitantes y en Cartagena con 500.000 habitantes.

Fueron registrados un total de 152 pacientes, siendo 34 de ellas del Instituto Colombiano de Cancerología de Bogotá, 108 del Hospital Universitario, San Vicente de Paúl de Medellín, y 10 del Hospital Universitario de Cartagena.

Para este total de 152 pacientes fueron tenidos en cuenta los casos diagnosticados entre los 60 días siguientes al parto pues lógicamente se supone que estas pacientes tenían la patología en cuestión, durante el embarazo, y en igual forma han procedido los autores que han escrito sobre este tema. En el post-parto fueron diagnosticados 10 en Bogotá, 45 en Medellín y 3 en Cartagena. 


\section{La Incidencia}

La incidencia de cáncer y embarazo fue relacionada al número total de canceres ginecológicos registrados en el mismo período de 10 años y así se aprecia que fue de $0.5 \%$ en Bogotá, de un total de 7.168 pacientes; $3.3 \%$ en $\mathrm{Me}$ dellín, de un total de 3.304 pacientes; y de $2.5 \%$ en Cartagena, de un total de 412 pacientes. En un total de 10.884 pacientes en Colombia se encontró en 152 lo que da una incidencia de $1.4 \%$.

Comparando esta cifra con la citada por Reed obtenida en Pondville State Cancer Hospital de un total de 15 casos en 853 pacientes que da un $1.7 \%$ (7), se aprecian que no son notorias las variaciones entre esta estadística de Estados Unidos y la de Colombia. Solo merece mencionarse la cifra alta de $3.3 \%$ de Medellín que tiene su origen, en la citología practicamente de rutina establecida en el post-parto a donde acuden siempre la paciente pues allí le enseñan y le ofrecen métodos para la planificación familiar, y las campañas del comité regional de lucha contra el cancer que emplea profusamente los diferentes medios de comunicación, para llegar a la comunidad.

Por ser los centros de Bogotá y MedeIlín lugares exclusivos de terapia oncológica, no fue posible valorar estas cifras en cuanto al número de partos, pero si pudo hacerse en Cartagena en donde fueron encontrados en los 10 años estudiados, 10 cánceres del cervix durante el embarazo de un total de 412 cánceres ginecológicos registrados y de 82.626 partos atendidos.

\section{La Edad}

La edad de la paciente, neoplasias interepiteliales y cánceres invasivos del cervix durante el embarazo fueron encontrados entre los 20 y los 50 años, es decir en la época de la nuvilidad femeni- na. Su mayor frecuencia correspondió entre los 30 y 34 años de edad y su incidencia guarda relación con una correcta curva de Gaus, tanto en Cartagena puerto maritimo, como en Medellín a 1.500 metros sobre el nivel del mar y en Bogotá a 2.400 metros de altura.

La mayor incidencia entre los $30 \mathrm{y}$ 34 años de esta patología en el embarazo, en Colombia, es la resultante de una serie de complejos fenómenos geográficos -zona ecuatorial como lo está casi toda latinoamerica - en donde la pubertad se inicia recién cumplidos los 10 años y la menarca llega a los 12 y en donde por razones culturales de comportamiento y por factores sociales y económicos las relaciones sexuales se inician en un alto porcentaje antes de cumplidos los 20 años, y es bien conocido el fenómeno epidemiológico de que el cáncer cervical se comporta como una enfermedad venérea.

\section{Edad Gestacional}

En el primer trimestre del embarazo fueron diagnosticados como carcinomas in situ 16 casos, lo que equivale a un $10 \%$ del total de los casos del presente trabajo, y como carcinoma invasor 18 casos que corresponden a un $12 \%$; es lógico suponer que en estos últimos casos la lesión se encontraba como tal desde antes de iniciar el embarazo y que ello no fue diagnosticado por falta de un examen adecuado o por no haber asistido la paciente a consulta médica previa. En el segundo trimestre del embarazo fueron diagnosticados como carcinoma in situ 16 casos lo que equivale a un $10 \%$ del total estudiado y como carcinoma invasor 19 casos que corresponde a un $13 \%$ del total estudiado. También en este segundo trimestre fue ligeramente superior la proporción de cánceres invasivos diagnosticados a la de cánceres insitu. 
En el tercer trimestre del embarazo fueron diagnosticados como carcinoma in situ 8 casos lo que equivale a un $5 \%$ del total de los casos, y como carcinoma invasor 16 casos que corresponde a un $10 \%$ del total estudiado. Si bien en este trimestre una tercera parte fue diagnosticado como neoplasia aún interepitelial, lo que indica procedimientos correctos y actualizados en la consulta prenatal, un considerable número mayor de casos que corresponden a las dos terceras partes de ellos, apenas se logró el diagnóstico en la etapa ya avanzada de carcinoma invasor $y$ se piensa ante estas cifras sino será que muchas pacientes acuden a la consulta prenatal muy tardiamente o si ellas visitan al gineco-obstetra solo si sienten alguna sintomatología como colpitis o hemorragias vaginales.

En los exámenes ginecológicos efectuados en los 60 días siguientes a la fecha del parto, fueron diagnosticados 44 casos de carcinoma in situ, lo que equivale a un $30 \%$ del total de casos; este alto porcentaje tiene su origen en la toma de citología cada vez más frecuente, en la revisión post-parto $y$ esta cifra aumentará mas aún cuando se incremen. te el uso del colposcopio en esta consulta.

Como carcinoma invasor del cervix se encontraron 15 de estos casos, es decir un $10 \%$ del total. Estas 15 pacientes fueron clasificadas, 5 de ellas como grado II, 9 como grado III y 1 como grado IV, lo que obviamente indica que su lesión podía ser diagnosticada clínicamente $o$ al menos sospechada en el momento del parto. En general los carcinomas invasores del cervix diagnosticados $y$ tratados en el post-parto tienen una posibilidad menor de curación y esa fue experiencia en los tres centros de Bogotá, Medellín y Cartagena.

\section{Los grados clínicos}

Mientras más alto sea el porcentaje de cánceres in situ diagnosticados en rela- ción a los cánceres invasores mayor será el adelanto científico en relación al diagnóstico de estas patologías cervicales en los lugares que son analizados.

Con los procedimientos diagnósticos mencionados, que son los usualmente empleados en Colombia en las consultas de los gineco-obstetrasen donde las tomas de muestras cervico-vaginales de citologías exfoliativas son cada vez más numerosas descle la década del 60, y en donde muy recientemente se ha implantado en unos pocos centros la práctica de los exámenes colposcópicos, se ha logrado ya un número superior de diagnóstico de neoplasias interepiteliales, de un $55 \%$, a los diagnósticos de cánceres invasores del cervix, de un $45 \%$ durante el embarazo y los 60 días siguientes al parto.

Los 68 cánceres invasores del cervix diagnosticados durante el embarazo y el post-parto en Colombia fueron definidos según la clasificación internacional de cáncer del cervix aprobada por la Federación Internacional de Ginecología y Obstetricia F.I.G.O. y se agruparon así: En el grado $\mathrm{I}-\mathrm{a}$, carcinoma microinvasivo un $4 \%$. En el grado I-b, estrictamente delimitado al cervix un $7 \%$. En el grado II-a, con extensión a vagina pero sin afección a los parametrios un $4 \%$. En el grado II-b con afección a los parametrios pero sin llegar a la pared pélvica un $14 \%$. En el grado III-a con afección vaginal hasta su tercio inferior un $2 \%$. En el grado III-b con afección total del parametrio hasta pared pélvica un $13 \%$. $Y$ en el grado IV con diseminación a los órganos adjuntos un $1 \%$.

Es oportuno mencionar en este momento que en Colombia como en tantos países de este y otros continentes, no se ha logrado dar una educación en el campo de la medicina preventiva orientada a la consulta de la futura madre al gineco-obstetra antes de iniciar un embarazo, como sería lo ideal. Se piensa que una recomendación dinámica de la 
F.I.G.O. en este sentido traería un gran beneficio a las comunidades del mundo.

Figura No. 1

NEOPLASIAS INTEREPITELIALES Y CARCINOMAS CERVICALES INVASIVOS EN EL EMBARAZO

\begin{tabular}{|c|c|c|c|}
\hline Total de Casos & 152 & Casos Post-Partum & 58 \\
\hline $\begin{array}{l}\text { Hospital Univ. San Vicente } \\
\text { de Paúl. Medell ín }\end{array}$ & 108 & & 45 \\
\hline $\begin{array}{l}\text { Instituto Nal. de } \\
\text { Cancerología } \\
\text { Bogotá. }\end{array}$ & 34 & & 10 \\
\hline $\begin{array}{l}\text { Hospital Univ. de } \\
\text { Cartagena. Cartagena. }\end{array}$ & 10 & & 3 \\
\hline
\end{tabular}

\section{TRATAMIENTO}

a. Tratamiento de las neoplasias interepiteliales y de los canceres invasivos del cervix.

En Colombia en los tres centros mencionados, las hiperplasias de las células de reserva, las displasias leves, moderadas y aún las severas del cervix, que pueden ser consideradas como neoplasias interepiteliales, no tienen tratamiento activo durante el embarazo y la conducta ante estos cambios epiteliales es la de permitir el parto por las vías naturales, y la de observación y vigilancia periódica y frecuente hasta los sesenta días después del parto, y si en esta fecha aún persisten imagenes citológicas o colposcópicas atípicas se continúan observando hasta los 180 días. Una de las razones para ello es la ya mencionada de que muchos de estos cambios histológicos regresan en forma precoz en los sesenta días siguientes al parto, $y$ en forma tardía hasta los ciento ochenta días. Luego el tratamiento será cuestionado por el porvenir obstétrico, y así si se desea otro hijo, se practicará un cono del cervix para definir diagnóstico y con intenciones de que este procedimiento quirúrgico sea terapéutico; y si no se desean más hijos se procederá a la histerectomía con conservación de anexos, pero con resección del tercio superior de vagina. b. El tratamiento de carcinoma in situ del cervix, durante el embarazo tiene también en los otros tres centros mencionados de Colombia, una terapéutica uniforme.

Cuando se diagnostica esta neoplasia interepitelial durante el embarazo y se tiene una razonable certeza de que no existen puntos donde ella sea microinvasora o francamente invasora, por medio de la biopsia múltiple o por medio del cono, se procede en forma igual a la expuesta anteriormente en la hiperplasia de las células de reserva o en las displasias leves, moderadas y severas; sea que el diagnóstico se efectúe en el primer, segundo o tercer trimestre del embarazo. Es decir, se procede después del diagnóstico de carcinoma in situ del cervix, a un control muy estricto cada dos semanas, para verificar que las imágenes citológicas y colposcópicas permanecen estáticas, y cuando así sucede se permite que el embarazo continúe su evolución hasta llegar a término y que se efectúe el parto por vía vaginal; ésta conducta de permitir el parto por vía natural se basa en el hecho de que no se ha encontrado que lesiones de carcinomas in situ se hayan vuelto carcinomas invasores cuando el parto ha sido atendido por vía vaginal $(8)$.

Figura No. 2

NEOPLASIAS INTEREPITELIALES Y CARCINOMAS CERVICALES INVASIVOS EN EL EMBARAZO: INCIDENCIA

\begin{tabular}{|lrrr|}
\hline Ciudad & $\%$ & Pacientes & $\begin{array}{r}\text { Canceres } \\
\text { Ginecológicos }\end{array}$ \\
\hline Bogotá & 0.5 & 34 & 7.168 \\
Medellín & 3.3 & 108 & 3.304 \\
Cartagena & 2.5 & 10 & 412 \\
Pondville & 1.7 & 15 & 853 \\
\hline
\end{tabular}

A los casos de carcinoma in situ del cervix seguidos durante el embarazo les es definida su conducta a las ocho sema- 
nas del parto, y esta conducta es igual a la de los casos diagnosticados por primera vez en el post-parto. En general el tratamiento es de histerectomía abdominal con conservación de anexos y resección del tercio superior de vagina.

Figura No. 3

NEOPLASIAS INTEREPITELIALES Y CARCINOMAS CERVICALES INVASORES EN EL EMBARAZO: EDAD GESTACIONAL

\begin{tabular}{|lrrcc|}
\hline Trimestre & $\begin{array}{c}\text { Casos } \\
\text { In Situ }\end{array}$ & $\%$ & $\begin{array}{c}\text { Casos } \\
\text { Invasivos }\end{array}$ & $\%$ \\
\hline Primero & 16 & 10 & 18 & 12 \\
Segundo & 16 & 10 & 19 & 13 \\
Tercero & 8 & 5 & 16 & 10 \\
Post-parto & 44 & 30 & 15 & 10 \\
\hline
\end{tabular}

Solo en casos muy excepcionales en donde a pesar de conocer la paciente su situación, desea un nuevo embarazo y es posible un control citológico y colposcópico estricto se procede a un cono como tratamiento con estudio histológico que verifique su total extirpación, y teniendo siempre muy presente la morbilidad post-quirúrgica y la morbilidad en el futuro embarazo. Recientemente para algunos de estos mismos excepcionales casos se ha empleado, más en Bogotá que en Medellín o en Cartagena, la criocirugía, que no permite el estudio histológico del tejido extirpado, y en donde solo pueden efectuarse para su control posterior la citología y la colposcopia.

\section{c. Tratamiento del carcinoma invasor} durante el embarazo.

Cuando se diagnostica un carcinoma invasor del cervix grado I-a o I-b, o grado II-a o II-b inicial, durante el primer o segundo trimestre del embarazo, se procede en forma uniforme en Colombia a efectuar la histerectomía con linfa adenectomía sin extraer el feto del útero. Se efectúa un estudio histológico del especimen y de los ganglios, y de acuerdo a ello se complementa o no con cobaltoterapia.

Cuando se diagnostica un carcinoma invasor del cervix, grado I-a o I-b, o un grado II-a o II-b inicial, durante el tercer trimestre del embarazo, el procedimiento elegido es la cesárea histerectomía con linfa adenectomía en el mismo acto quirúrgico, que tiende a sangrar un poco más de lo habitual. El acto quirúrgico se hace cuando los estudios ecográficos y del líquido amniótico indican que el feto ya es viable y cuando los índices de maduración pulmonar se retardan en estos casos pueden emplearse corticoides para tal fin. En uno de los centros se efectúa la cesárea simple y dos semanas después la histerectomía con linfaadenectomía. En todos los casos comentados se procede al estudio histológico del material quirúrgico y de acuerdo al informe del patólogo se procede a la cobaltoterapia complementaria.

En los grados II-b avanzados, en los casos III-a y III-b, y IV-a diagnosticados en el primer $y$ en el segundo trimestre del embarazo, el tratamiento se iniciará con cobaltoterapia que producirá a las pocas semanas el aborto espontáneo y luego se complementará con radium intracavitario.

Figura No. 4

CARCINOMAS CERVICALES INVASORES EN EL

EMBARAZO CLASIFICACION CLINICA DE LA F.I.G.O.

\begin{tabular}{|c|c|}
\hline Casos totales & 68 \\
\hline | a....... $4 \%$ & $111 \mathrm{a} \ldots \ldots \ldots 2 \%$ \\
\hline I b....... $7 \%$ & III b $\ldots, \ldots \ldots 13 \%$ \\
\hline $11 a \ldots \ldots \ldots 4 \%$ & IV $\ldots \ldots \ldots 1 \%$ \\
\hline II b....... $14 \%$ & \\
\hline
\end{tabular}

En los mismos grados II-b avanzados, y en los casos III-a y III-b, y IV-a, diagnosticados en el tercer trimestre del embarazo, cuando se compruebe madurez fetal por ecografía y estudio del líquido 
amniótico, se practicará cesárea corporal y posterior tratamiento con cobalto y radium.

Los cánceres invasivos del cervix diagnosticados en el post parto se tratan con los mismos principios o normas de los casos de pacientes no embarazadas, pero su pronóstico es menos favorable, y así ha sido observado en Colombia; da la impresión de que los cambios anatómicos del embarazo y la gran variación de los esteroides sexuales influyen en un sentido desfavorable para lograr su total curación.

\section{RESUMEN}

Se hacen algunas consideraciones sobre la importancia de la citología, de la colposcopia, de la biopsia múltiple y del cono cervical, como elementos para el diagnóstico y el pronóstico de las neoplasias interepiteliales y de los carcinomas invasores del cervix uterino, durante el embarazo y el post-parto. Se analizan los resultados de 152 casos de esta patología en 10 años de estudio de acuerdo a su incidencia, su edad gestacional y los grados clínicos. Se delimitan conductas terapeúticas en las lesiones neoplásicas interepiteliales y en los cánceres invasores del cervix durante el embarazo.

\section{DIAGNOSIS AND TREATMENT}

\section{OF INTER-EPITHELIAL NEOPLASIAS AND INVASIVE CERVICAL} CARCINOMAS DURING PREGNANCY

\section{Summary}

This article emphazises some considerations regarding the importance of citology, colposcopy, multiple biopsy and cervical cone, as elements for the diagnosis and prognosis of inter-epithelial neoplasias and invasive carcinomas of the uterine cervix during pregnancy and postpartum. 152 cases with this pathology were studied during ten years, especially regarding incidence, clinical degree and gestational age.

The authors also present indications for therapy of interepithelial neoplasias and invasive carcinomas of the cervix during pregnancy.

\section{BIBLIOGRAFIA}

1. TAKAHASHI, M. (1973). Atlas en color de citología del cancer. P. 30 Editors: Científico Médica. Barcelona.

2. JIMENEZ, M. NOGALES, F. (1977). Citopatología Ginecológica. P. 123-169 Editors: Científico Médica. Barcelona.

3. ISHIZUKA, Y. OOTA, K. MASUBUCHI, K. (1975) Citodiagnóstico Práctico. P. 66-104 Editors: Científico Médica. Barcelona.

4. BONILLA-MUSOLES, F. (1978). EI Cuello uterino y sus enfermedades. P. 37-65 Editors: Jims. Barcelona

5. URIBE, J. DIAZ, R. RODRIGUEZ, L. (1978). Estudio estadístico de lesiones premalignas de cuello. Rev. Col. de Obs. v Gin. P. 119-138. Vol. 29.

6. RODRIGUEZ, M. (1979). Carcinoma uterino en embarazadas. Hosp. Universitario San Ignacio. Universidad Pontificia Javeriana. Bogotá.

7. GREEN, T.H. Jr. (1974). Carcinoma of the cervix in pregnancy from surgical desease in pregnancy barber H. Graber E. Editors: Saunders Co. Philadelphia.

8. D.E ALVAREZ, R.R. (1977). Texbook of Gynecology. P. 271. Editors: De Alvarez Philadelphia. 